\title{
Reduced intensity conditioning increases risk of severe cGVHD: identification of risk factors for cGVHD in a multicenter setting
}

\author{
Gabriel Afram ${ }^{1}\left[\right.$. Jose Antonio Pérez Simón ${ }^{2} \cdot$ Mats Remberger $^{3} \cdot$ Teresa Caballero-Velázquez $^{2} \cdot$ Rodrigo Martino $^{4}$. \\ Jose Luis Piñana ${ }^{4,6}$. Olle Ringden ${ }^{3}$. Albert Esquirol ${ }^{4}$. Lucia Lopez-Corral ${ }^{5}$. Irene Garcia ${ }^{4}$. Oriana López-Godino ${ }^{5}$. \\ Jordi Sierra ${ }^{4} \cdot$ Dolores Caballero $^{5} \cdot$ Per Ljungman $^{1} \cdot$ Lourdes Vazquez $^{5} \cdot$ Hans Hägglund $^{7}$
}

Received: 27 January 2018 / Accepted: 5 April 2018 / Published online: 25 April 2018

(c) The Author(s) 2018

\begin{abstract}
Chronic graft-versus-host disease (cGVHD) remains a major cause of morbidity and mortality after allogeneic hematopoietic stem cell transplantation (HSCT). Aim is to identify risk factors for the development of cGVHD in a multicenter setting. Patients transplanted between 2000 and 2006 were analyzed $(n=820)$. Donors were HLA-identical siblings $(57 \%)$, matched unrelated donors (30\%), and HLA-A, B or DR antigen mismatched (13\%). Reduced intensity conditioning (RIC) was given to $65 \%$ of patients. Overall incidence of cGVHD was $46 \%$ for patients surviving more than 100 days after HSCT $(n=747)$. Older patient age [HR 1.15, $p<0.001]$, prior acute GVHD [1.30, $p=0.024]$, and RIC [1.36, $p=0.028]$ increased overall cGVHD. In addition, RIC [4.85, $p<0.001]$, prior aGVHD [2.14, $p=0.001]$ and female donor to male recipient [1.80, $p=0.008]$ increased the risk of severe cGVHD. ATG had a protective effect for both overall [0.41, $p<0.001]$ and severe cGVHD [0.20, $p<0.001]$. Relapse-free survival (RFS) was impaired in patients with severe cGVHD. RIC, prior aGVHD, and female-to-male donation increase the risk of severe cGVHD. ATG reduces the risk of all grades of cGVHD without hampering RFS. GVHD prophylaxis may be tailored according to the risk profile of patients.
\end{abstract}

Keywords ATG · Graft-versus-host disease (GVHD) · Risk factor

Gabriel Afram

gabriel.afram@ki.se

1 Department of Hematology, Karolinska University Hospital Huddinge, Stockholm, Sweden

2 Department of Hematology, Instituto de Biomedicina de Sevilla (IBIS), Hospital Universitario Virgen del Rocío/CSIC/Universidad de Sevilla, Seville, Spain

3 Centre for Allogeneic Stem Cell Transplantation, Karolinska University Hospital Huddinge, Stockholm, Sweden

4 Department of Hematology, Hospital de la Santa Creu i Sant Pau, Barcelona, Spain

5 Department of Hematology, Hospital Universitario de Salamanca-IBSAL, Salamanca, Spain

6 Department of Hematology, Hospital Clinico Universitario, Valencia, Spain

7 Division of Hematology, Department of Medical Sciences, Uppsala University Hospital, Uppsala, Sweden

\section{Introduction}

Chronic graft-versus-host disease (cGVHD) remains one of the most severe complications after allogeneic hematopoietic stem cell transplantation (HSCT), affecting both the quality of life and mortality of long-term survivors [1-4]. Its impact on morbidity and mortality varies depending on the severity and number of organs involved, allowing the classification of patients into mild, moderate, and severe cGVHD according to the NIH, and identifying those at low, intermediate, or high risk of developing GVHD-related morbidity and mortality. Chronic GVHD is associated with a graft-versustumor effect (GVT) that decreases the risk of relapse after transplant [5]. These findings emphasize the importance of appropriate management of cGVHD, which should be individualized according to the patients' characteristics.

Risk factors for cGVHD include high recipient age, prior acute GVHD, female donor to male recipient [6], HLA disparity between recipient and donor, and use of peripheral blood as a source of progenitor cells [7-9]. CGVHD is an increasingly frequent complication after HSCT due, at least 
in part, to the more frequent use of peripheral blood stem cells, higher age of recipients/donors, and increased use of mismatched and unrelated donors. Recently, we retrospectively classified a large cohort of patients in terms of cGvHD subtype and severity according to the NIH proposal [10]. To the best of our knowledge, there are no data on cGvHD risk factor analysis based on the cGvHD NIH classification.

Various studies have attempted to identify the best strategy to prevent GVHD, and to date, only the use of in vitro or in vivo $\mathrm{T}$ cell depletion has been shown to reduce the risk of cGVHD, although its impact on survival has been relatively limited in unselected series of patients [11]. Therefore, the prophylaxis regimen should be tailored on the basis of individual patient and transplant characteristics, and more effective immunosuppressive strategies could benefit patients at high risk of severe forms of cGVHD and low risk of relapse, while the contrary could apply to patients at high risk of relapse in the event of displaying a lower risk of cGVHD.

Our study aimed to highlight risk factors for developing mild, moderate, and severe cGVHD in a multicenter setting. We retrospectively included 747 patients who had undergone HSCT between the years 2000-2006.

\section{Patients and methods}

Eight hundred and twenty patients undergoing HSCT at three different centers from January 2000 to December 2006 were included in this retrospective study. The analysis was restricted to those patients surviving more than 100 days after HSCT $(n=747)$. The number of patients included from each center was: Karolinska $(n=425)$, Salamanca $(n=162)$ and Sant Pau $(n=160)$. The study protocol was approved by the regional ethics committees (Regionala Etikprövningsnämnden, Stockholm and Comité Etico CEIM (Comité de ética de la investigación con medicamentos) for Spain and was performed in accordance with the Declaration of Helsinki. Chronic GVHD was retrospectively categorized according to the NIH consensus criteria [12]. The patients' characteristics are summarized in Table 1.

The median age at the time of transplantation was 44 years $(<1-70)$. The most common diagnosis was acute leukemia (acute myeloid leukemia or acute lymphoblastic leukemia) in 34\% of patients; chronic leukemia was diagnosed in $15 \%$ and lymphoma in $14 \%$.

\section{Donors and stem cell source}

Fifty-seven percent of patients received sibling donor grafts, $13 \%$ received grafts with one HLA-A, B, or DR antigen or allele mismatch, and 30\% received grafts from HLA-A, B, and DR-matched unrelated donors. All patients and donors were typed using PCR-SSP high-resolution typing for both
HLA class I and II alleles [13]. Seventy-six percent received peripheral blood hematopoietic stem cells (PBSC) from G-CSF stimulated donors, $20 \%$ received bone marrow grafts, and $4 \%$ received cord blood grafts.

\section{Conditioning}

Myeloablative regimens were used in 34\% of cases, the rest receiving reduced intensity conditioning (RIC). Forty-four percent received in vivo $\mathrm{T}$ cell depletion with anti-thymocyte globulin (ATG, Thymoglobulin, Genzyme, Cambridge, MA, USA) $(n=295)$ or Campath ${ }^{\circledR}(n=30)$. Patients at Karolinska (center $A$ ) received ATG (Thymoglobulin, Genzyme, Cambridge, MA, USA) at a dose of $4-8 \mathrm{mg} / \mathrm{kg}$. Patients from Salamanca and Sant Pau (centers $B$ and $C$ ) received ATG at a dose of $7.5 \mathrm{mg} / \mathrm{kg}$. ATG was administered for 3-4 days with the last dose given on day 1 or 2. ATG was administered to patients with unrelated donors, HLA-mismatched donors, and those with non-malignant diseases.

\section{GVHD prophylaxis and treatment}

Most patients received GVHD prophylaxis with cyclosporine A or tacrolimus combined with methotrexate (80\%), while $11 \%$ received mycophenolate mofetil instead of methotrexate. The remaining $9 \%$ of patients received $\mathrm{CyA}$ or tacrolimus combined with prednisone or other immunosuppressive regimes.

First-line treatment for cGVHD was based on cyclosporine A or tacrolimus plus prednisone. Disease response was generally evaluated 5 weeks after initiation of treatment and subsequently every 3 months until cessation of treatment.

All patients received antibacterial, antifungal, and antiviral prophylaxis according to standard protocols at each center.

\section{Statistical analysis}

The incidence of chronic GVHD was estimated using an estimator of cumulative incidence curves. Patients were censored at the time of death or last follow-up. Only patients surviving more than 100 days after HSCT were included in the analysis and the competing event was death without chronic GVHD. Categorical parameters were compared using Chi-square test and continuous variables were compared using the Mann-Whitney test. Multivariate predictive analyses were performed using Gray's test and the proportional sub-distribution hazard regression model of Fine and Gray [14]. A stepwise backward procedure was used to construct a set of independent predictors. All predictors with a $p$ value below 0.10 in the univariate analysis were introduced into the multivariate model and sequentially removed if the 
Table 1 Characteristics of HSCT patients with or without chronic GVHD

\begin{tabular}{|c|c|c|c|}
\hline Factor & No cGVHD & cGVHD & $p$ value \\
\hline$N=$ & 409 & 338 & \\
\hline Age & $37(<1-69)$ & $51(<1-70)$ & $<0.001$ \\
\hline Donor age & $38(0-74)$ & $45(0-77)$ & $<0.001$ \\
\hline Sex (male/female) & $241 / 168$ & $214 / 124$ & 0.25 \\
\hline Donor sex (male/female) & $239 / 164$ & $179 / 152$ & 0.18 \\
\hline Female to male & $76(19 \%)$ & $89(26 \%)$ & 0.013 \\
\hline Disease stage (early/late) & $180 / 199$ & $129 / 195$ & 0.04 \\
\hline Stem cell source $(\mathrm{PBSC} / \mathrm{BM} / \mathrm{CB})$ & $278 / 108 / 23$ & $290 / 43 / 5$ & $<0.001$ \\
\hline $\mathrm{CD} 34+$ cell dose $\left(\times 10^{6} / \mathrm{kg}\right)$ & $6.8(0.1-68)$ & $6.7(0.1$ to -19.9$)$ & 0.89 \\
\hline \multicolumn{4}{|l|}{ Donor } \\
\hline Sibling & $196(48 \%)$ & $242(72 \%)$ & \multirow[t]{2}{*}{$<0.001$} \\
\hline MUD & $153(37 \%)$ & $64(19 \%)$ & \\
\hline Mismatched & $60(15 \%)$ & $31(9 \%)$ & 0.03 \\
\hline \multicolumn{4}{|l|}{ Conditioning } \\
\hline MAC & $165(43 \%)$ & $85(26 \%)$ & \multirow[t]{3}{*}{$<0.001$} \\
\hline RIC & $232(57 \%)$ & $245(74 \%)$ & \\
\hline ATG & $233(57 \%)$ & $92(27 \%)$ & \\
\hline \multicolumn{4}{|l|}{ Diagnosis } \\
\hline Non-malignant & $53(13 \%)$ & $17(5 \%)$ & $<0.001$ \\
\hline AML/ALL & $90 / 52(35 \%)$ & $81 / 31(33 \%)$ & Ns \\
\hline CML/CLL & $41 / 13(13 \%)$ & $36 / 21(17 \%)$ & Ns \\
\hline Lymphoma & $55(13 \%)$ & $53(16 \%)$ & Ns \\
\hline MDS/MPS & $36(9 \%)$ & $40(12 \%)$ & Ns \\
\hline Myeloma & $29(7 \%)$ & $30(9 \%)$ & Ns \\
\hline Solid tumor & $30(7 \%)$ & $13(4 \%)$ & Ns \\
\hline Other & $10(2 \%)$ & $16(5 \%)$ & Ns \\
\hline
\end{tabular}

Early stage $\mathrm{CR} 1 / \mathrm{CP} 1$, Late stage beyond CR1/CP1, PBSC peripheral blood stem cells, $B M$ bone marrow, $C B$ cord blood, $M U D$ matched unrelated donor, $M A C$ myeloablative conditioning, $R I C$ reduced intensity conditioning, $A T G$ anti-thymocyte globulin, $A M L$ acute myeloid leukemia, $A L L$ acute lymphoid leukemia, $C M L$ chronic myeloid leukemia, $C L L$ chronic lymphoid leukemia, MDS myelodysplastic syndrome, MPS myeloproliferative syndrome $p$ value was above 0.05 . Risk factors included in the univariate analysis were: patient and donor age, patient and donor sex, sex mismatch, disease stage, stem cell source, donor type, conditioning, ATG, diagnosis, center, GVHD prophylaxis, CD34 + cell dose and prior aGVHD. All tests were two-sided. The type I error rate was fixed at 0.05 for factors potentially associated with time-to-event outcomes. Analyses were performed using the cmprsk package (developed by Gray, June 2001), S-PLUS 6.2 software and Statistica software.

\section{Results}

The overall cumulative incidence of acute GVHD was $56 \%$ and that of cGVHD was $48 \%$ (95\% CI 44-52\%). The incidence of $\mathrm{cGVHD}$ at the three centers $\mathrm{A}, \mathrm{B}$, and $\mathrm{C}$ was $32 \%(27-37 \%), 70 \%$ (63-77\%), and 58\% (50-66\%), respectively. The percentage of children transplanted at each center was 29, 6, and 0\%, respectively, for center A, B, and C. Median time to onset for cGVHD was 5.7 (2.0-77) months post-transplant.

ATG was administered at a frequency of 71,13 , and $1 \%$ in the three centers, respectively. The percentage of sibling donor transplants at each center was 42,79 , and $81 \%$, respectively. Results were similar in terms of survival at the three centers [53\% (48-58\%), 49\% (41-57\%), 52\% (45-59\%), respectively] as well as in terms of relapse-free survival (RFS) [46\% (41-51\%), 39\% (31-47\%), 42\% (35-49\%)] at 5 years.

\section{Risk factors for overall cGVHD}

In univariate analysis, significant risk factors for the development of cGVHD included prior acute GVHD, RIC, PBSC, sex mismatch, increased donor age, sibling donor, and late disease stage (beyond first remission). The use of ATG was a protective factor (Fig. 1). In multivariate 


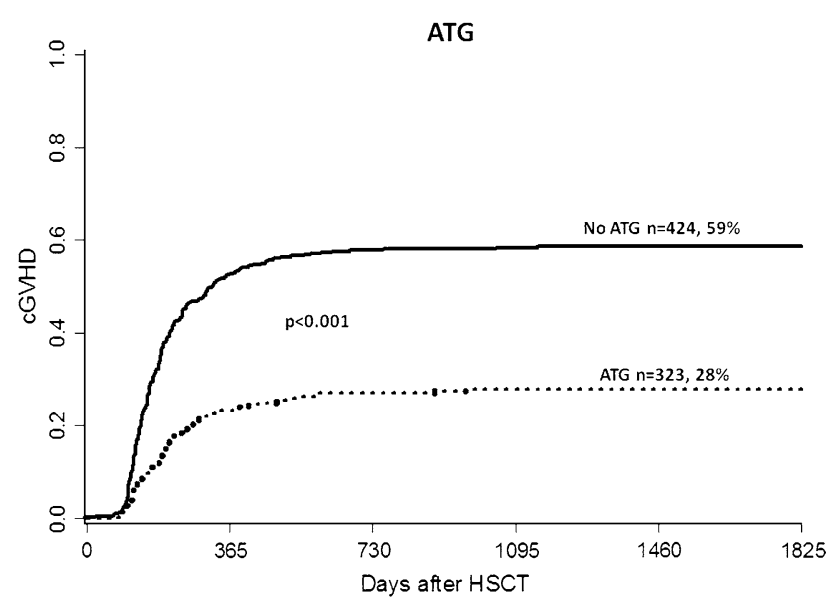

Fig. 1 Chronic GVHD incidence in patients treated with anti-thymocyte globulin (ATG) versus no ATG

analysis, the following variables significantly influenced the risk of overall cGVHD: use of ATG [HR $=0.41,95 \%$ CI $(0.32-0.52), p<0.001]$, higher patient age (in 10-year increments) $[\mathrm{HR}=1.15,95 \% \mathrm{CI}(1.07-1.24), p<0.001]$, prior acute GVHD [HR $=1.30,95 \%$ CI $(1.04-1.63), p=0.024]$ and reduced intensity conditioning (RIC) $[\mathrm{HR}=1.36,95 \%$ CI (1.04-1.79), $p=0.028]$. When analyzing risk factors for cGVHD after correcting for differences between patients receiving RIC or MAC, it is apparent that RIC patients still have higher cGVHD incidence $[\mathrm{HR}=1.38,95 \% \mathrm{CI}$ $(1.02-1.88), p=0.038]$. The analysis was done in order to eliminate confounding factors in each group.

\section{Risk factors for severe cGVHD}

The overall incidence of severe cGVHD was $14 \%$ (95\% CI 11-17\%). In multivariate analysis, female donor to male recipient [HR 1.80 (95\% CI 1.17-2.78), $p=0.008$ ], RIC [HR 4.85 (95\% CI 2.40-9.83) $p<0.001$ ], and prior aGVHD [HR 2.14 (95\% CI 1.34-3.42), $p=0.001$ ] significantly increased the risk of severe $\mathrm{GGVHD}$ while ATG had a protective effect [HR 0.20, (95\% CI 0.11-0.37), $p<0.001]$.

Based on our findings, we developed a scoring system including significant risk factors from multivariate analysis with regard to severe cGVHD incidence (Fig. 2). According to this scoring system, the risk of developing severe cGVHD was $3.1,6.8,26.4$, and $40.4 \%$ at 5 years post-transplant among patients with one, two, three, or four risk factors.

Next, we conducted a multivariate analysis including only significant risk factors present at the time of transplantation. In this analysis, we found that patient age $>45$ years, female-to-male donation, and RIC increased the risk of severe cGVHD, while ATG remained a protective factor. When two to three of these risk factors were present, there

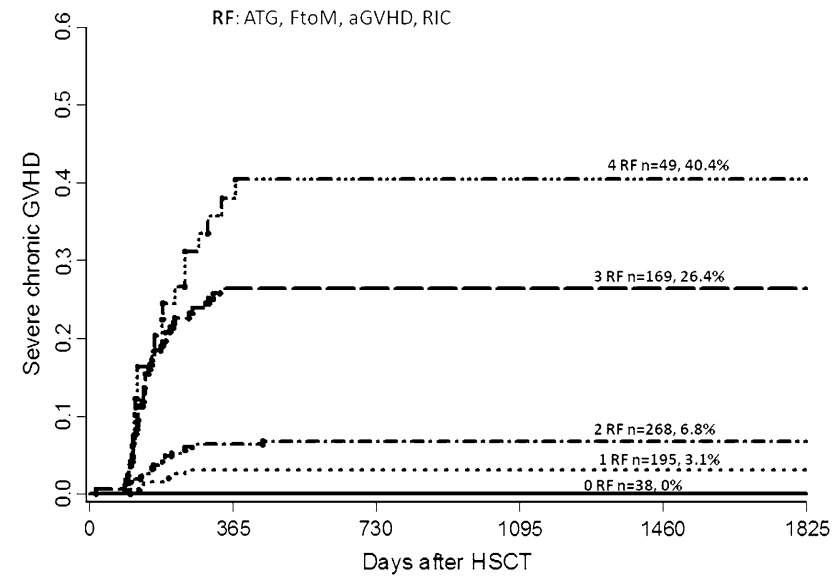

Fig. 2 Risk factor score for developing severe cGVHD including risk factors from multivariate analysis with female donor to male recipient, reduced intensity conditioning (RIC), anti-thymocyte globulin (ATG) and prior acute GVHD

Table 2 Incidence of severe chronic GVHD after HSCT depending on number of risk factors (RF) and inclusion of anti-thymocyte globulin (ATG) or not in the conditioning therapy. Only factors known at time of transplantation were analyzed

\begin{tabular}{llcl}
\hline Severe cGVHD & ATG $(\%)$ & No ATG $(\%)$ & $p$ value \\
\hline $1 \mathrm{RF}$ & 4 & 6 & NS \\
$2 \mathrm{RF}$ & 4 & 24 & $<0.001$ \\
$3 \mathrm{RF}$ & 7 & 40 & 0.01 \\
\hline
\end{tabular}

Table 3 Overall survival (OS) and transplant-related mortality (TRM) 5 years after HSCT, depending on the severity of chronic GVHD (95\% confidence interval given in brackets)

\begin{tabular}{lll}
\hline Grade of cGVHD & TRM & OS \\
\hline No cGVHD & $24 \%(19-29 \%)$ & $51 \%(46-56 \%)$ \\
Mild & $14 \%(8-20 \%)$ & $72 \%(63-81 \%)$ \\
Moderate & $18 \%(11-25 \%)$ & $71 \%(63-79 \%)$ \\
Severe & $31 \%(21-41 \%)$ & $50 \%(39-61 \%)$ \\
$p$ value & $<0.001$ & $<0.001$ \\
All patients & $22 \%(19-25 \%)$ & $57 \%(53-61 \%)$ \\
\hline
\end{tabular}

was a significant protective effect from ATG on the incidence of severe cGVHD at 5 years post-transplant (Table 2).

\section{Transplant-related mortality (TRM), overall, and relapse-free survival}

Concerning OS, the worst outcome was seen in patients with severe cGVHD or no cGVHD. Similar results were observed concerning TRM. In this analysis, we found that the best outcomes were obtained in patients who developed mild-moderate cGVHD (Table 3). 
Relapse-free survival at 5 years was similar in patients developing mild or moderate cGVHD [59\% (49-59\%) vs. 64\% (55-73\%), respectively], but significantly higher in these same groups compared to patients without cGVHD or with severe cGVHD [RFS of 39\% (34-44\%) and 46\% (35-57\%), respectively; $p<0.001$ for mild and moderate compared to severe or no cGVHD] (Fig. 3). ATG had no influence on RFS (Fig. 4).

The risk of relapse or death was increased among patients with high-risk disease at transplantation $[\mathrm{HR}=1.61,(95 \%$ CI 1.29-2.01), $p<0.001]$. Interestingly, the best RFS was observed in patients with mild-to-moderate cGVHD irrespective of disease stage at time of transplantation (Table 4).

\section{Discussion}

Chronic GVHD remains the major cause of morbidity and mortality in long-term survivors after allogeneic stem cell transplantation. However, it is also correlated with a strong graft-versus-tumor effect. Thus, proper management of patients should be based on individualized strategies taking into account the notion that the absence of cGVHD might hamper relapse-free survival while severe cGVHD leads to increased mortality due to infectious complications and organ failure [15-18].

In the current study, we identified risk factors with regard to developing severe cGVHD, including female donor-tomale donor recipient, prior aGVHD and use of reduced intensity conditioning. While the first two risk factors have previously been described, the latter requires further clarification. In this regard, the finding of a higher risk of cGVHD among patients receiving RIC is somewhat surprising, since previous studies have not been able to show a difference in terms of cGVHD incidence depending on the type of

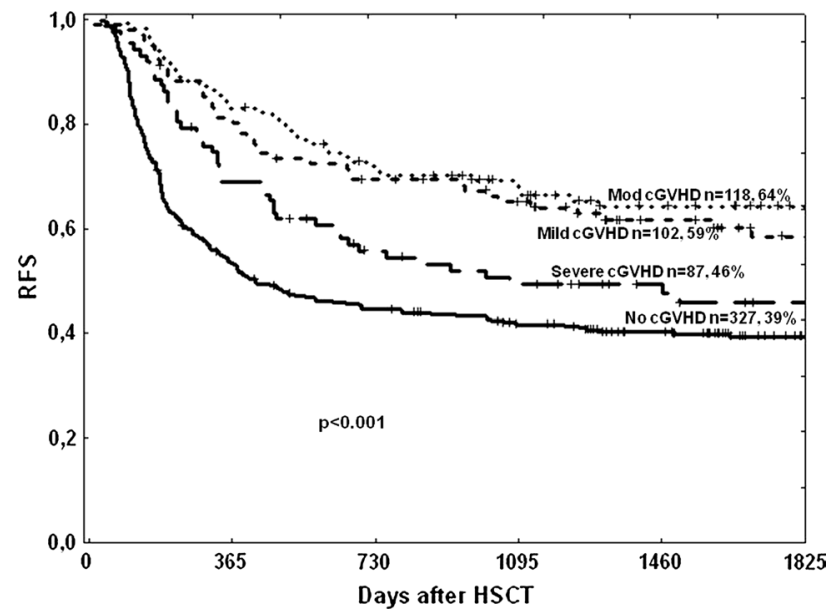

Fig. 3 Relapse-free survival dependent on severity of chronic GVHD

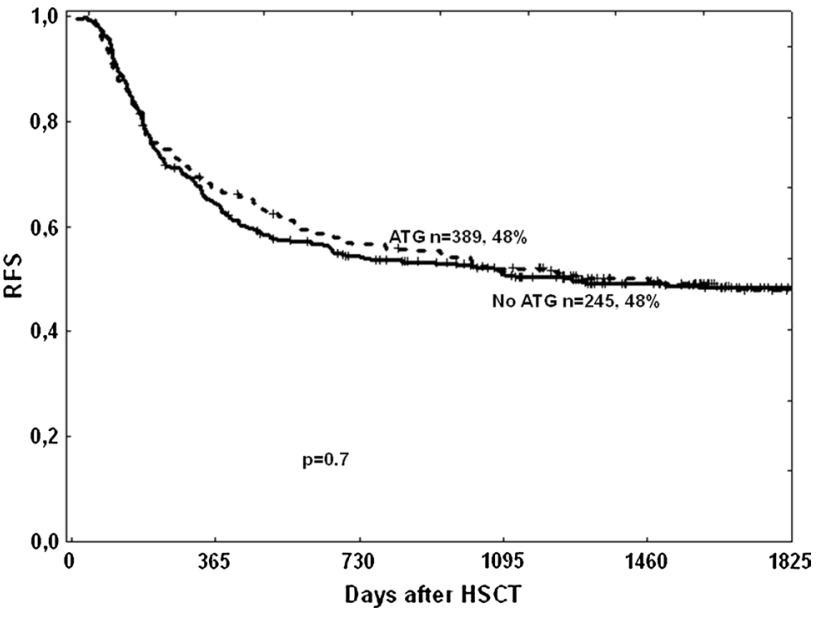

Fig. 4 Relapse-free survival in patients conditioned with or without anti-thymocyte globulin (ATG)

conditioning regimen $[19,20]$. Mechanisms involved in the development of acute and chronic GVHD are not entirely congruent. In this regard, cGVHD is not simply the end stage of acute GVHD [21]. In accordance with this hypothesis, use of RIC might, in fact, decrease the risk of acute and increase the risk of chronic GVHD. It could be speculated that acute GVHD is mostly dependent on the cytokine storm mediated by the tissue injury induced by the high doses of chemoradiotherapy, which is avoided in the RIC setting. On the other hand, chronic GVHD would be more dependent on the persistence of host-origin antigen-presenting cells that might trigger an alloresponse in donor $\mathrm{T}$ cells. The use of RIC favors the persistence of a mixed chimerism for a longer period post-transplant as compared to myeloablative conditioning. It could also be argued that since many centers show a preference toward treating older patients with RIC mainly due to comorbidities, the median age in this patient group is higher compared to those treated with myeloablative conditioning (MAC). This is also true in our study, and therefore, RIC patients are a higher-risk subpopulation for the development of cGVHD compared to MAC.

Table 4 Relapse-free survival (RFS) in different grades of chronic GVHD according to disease stage

\begin{tabular}{lllc}
\hline Five-year RFS & $\begin{array}{l}\text { Early disease } \\
(\%)\end{array}$ & $\begin{array}{l}\text { Late disease } \\
(\%)\end{array}$ & $p$ value \\
\hline No cGVHD & 45 & 36 & 0.09 \\
Mild & 72 & 48 & 0.016 \\
Moderate & 83 & 53 & $<0.001$ \\
Severe & 49 & 44 & 0.80 \\
\hline
\end{tabular}

Early stage $\mathrm{CR} 1 / \mathrm{CP} 1$, Late stage beyond CR1/CP1 
In the present study, we have shown that the addition of ATG decreases the overall incidence of cGVHD without hampering relapse-free survival, which is consistent with previous studies [6, 11, 17, 22-25].

Langston et al. showed that the addition of ATG to fludarabine-melphalan in the RIC regimen for patients receiving partially matched URD transplants does not increase the rates of relapse or infection. The same study did not show any difference with regards to cGVHD incidence in the two groups with Fu-Mel-ATG versus Flu-Mel [26]. One recent report shows that a fludarabine-containing $\mathrm{RIC}$ regimen with the addition of ATG is effective and safe for adults up to the age of 69 [27]. Investigation of the effect of ATG in a matched related donor myeloablative setting has shown beneficial effects with reduction in both severe aGVHD and cGVHD, which translated into higher survival [28].

Nevertheless, the use of pretransplant ATG as GVHD prophylaxis in patients receiving grafts from URD has been discussed for years, and although most studies have shown a significant reduction in acute GVHD and cGVHD, they have lacked evidence concerning a potential benefit on overall survival [11, 13, 24, 25, 29]. A GITMO group showed a significant reduction in the incidence of grades III-IV aGVHD and extensive cGVHD, but this positive effect was counterbalanced by the increased risk of infections. In a follow-up analysis of this study, encouraging results included a reduced risk of chronic lung dysfunction, protection against extensive chronic GVHD, and an improved quality of life [21]. In this prospective setting, there was no significant difference between the two groups regarding relapse and long-term survival.

These data contrast with a recent report from CIBMTR describing lower overall survival, lower disease-free survival, and an increased risk of relapse in patients receiving in vivo T-cell depletion [30]. This difference might be due to the heterogeneous nature of the population included in the CIBMTR study, in which patients received different preparations of ATG, dosage, conditioning regimens and donor types. In addition, the type of conditioning determines the effect of the antibody therapy since, according to Soiffer et al., there is a deleterious effect of in vivo $\mathrm{T}$ cell depletion among patients undergoing RIC. Furthermore, both the dosage and the preparation of ATG seem to be important in terms of transplant outcomes [13, 24, 25, 31]. In this regard, most studies have shown that an intermediate dose of rabbitATG (Thymoglobulin, Genzyme) ranging from 5 to $8 \mathrm{mg} / \mathrm{kg}$ leads to a positive reduction in severe aGVHD and cGVHD without impairing relapse or long-term survival.

In addition, in the current study, we show that increasing patient age significantly influences the incidence of cGVHD, but only up to 50 years, which is consistent with a recent study including 206 patients who underwent RIC allo-HSCT plus $5 \mathrm{mg} / \mathrm{kg}$-ATG. In this study, there was no difference in the incidence of extensive cGVHD in patient groups younger or older than 60 years [32].

Most importantly, the current study allowed us to develop a scoring system with clearly identifiable subgroups of patients at different risk of developing overall or severe cGVHD. Remarkably, both severe cGVHD and no cGVHD had a similar adverse impact on outcome. Based on these data, mild or moderate cGVHD show a desirable GvL effect without increasing the mortality from the procedure. Interestingly we found that in patients with more advanced disease stages the difference in RFS became less prominent, perhaps suggesting that this patient group relapses before a significant GvL effect is obtained. All together, these data suggest that patients at higher risk of severe cGVHD should be identified in order to have a favorable influence on longterm outcomes via the use of more intense immunosuppressive strategies, such as the use of ATG. In this regard, the lack of conclusive data regarding the impact of the use of ATG on survival in most previously reported randomized studies could be attributed to the lack of selection criteria, such that, according to our data, older males with female donors receiving RIC transplants would benefit the most from receiving this prophylaxis. Pretransplant identification of patients at risk of developing graft-versus-host disease remains an unmet medical need. In this regard, even among patients receiving transplantation from HLA-identical donors, the risk of GVHD varies greatly among individuals. Recently, Sorror et al. [33] reported that the pretransplant comorbidity index predicts the risk of severe aGVHD and subsequent mortality. In the current study, we developed a scoring system for cGVHD including risk factors known at the time of transplant: RIC, female-to-male donation and patient age $>45$ years. Patients with these three risk factors had an incidence of severe cGHD of $40 \%$ at 5 years, while the same patients had a cumulative incidence of $7 \%$ at 5 years when they received ATG. Accordingly, the scoring system allows the tailoring of strategies to prevent cGVHD in the early post-transplant period and in the long-term follow-up. Furthermore, we also identified RIC, female-tomale donation, prior acute GVHD and not receiving ATG as GVHD prophylaxis as risk factors for developing severe cGVHD. This information would be of the greatest use for modifying immunosuppression during the post-transplant follow-up period. In this regard, these patients should be carefully monitored and might benefit from receiving early treatment once signs or symptoms of cGVHD appear.

In conclusion, in the current study, we identified subgroups of patients with different risk of cGVHD; these parameters might help to tailor GVHD prophylaxis and manage immunosuppression in the long term.

Author's contribution GA and $\mathrm{HH}$ designed the study, wrote first draft, and collected data for the Swedish patient cohort. JAPS contributed as 
second author. MR and RM conducted statistical analysis. TC-V and OLG conducted data collection for Spanish cohort. DC, LLC and LV conducted clinical management and critical review for patients added from Salamanca. JS conducted data collection and clinical management together with JLP, IG and AE for patients from Barcelona. OR is professor at the HSCT unit at Karolinska Huddinge and contributed with experience-based input. PL was at the time head of the department of Hematology and contributed with experience-based input.

\section{Compliance with ethical standards}

Conflict of interest All authors declare that they have no conflict of interest.

Open Access This article is distributed under the terms of the Creative Commons Attribution 4.0 International License (http://creativecommons.org/licenses/by/4.0/), which permits unrestricted use, distribution, and reproduction in any medium, provided you give appropriate credit to the original author(s) and the source, provide a link to the Creative Commons license, and indicate if changes were made.

\section{References}

1. Lee S. New approaches for preventing and treating chronic graftversus-host disease. Blood. 2005;105(11):4200-6.

2. Shulman HM, Sullivan KM, Weiden PL, McDonald GB, Striker GE, Sale GE, et al. Chronic-graft-versus-host syndrome in man: a long-term clinicopathologic study of 20 Seattle patients. Am J Med. 1980;69:204-17.

3. Thomas E, Storb R, Clift RA, Fefer A, Johnson FL, Neiman PE, et al. Bone marrow transplantation (first of two parts). N Engl J Med. 1975;17(292):832-43.

4. Thomas E, Storb R, Clift RA, Fefer A, Johnson FL, Neiman PE, et al. Bone-marrow transplantation (second of two parts). N Engl J Med. 1975;24(292):895-902.

5. Storb R, Gyurkocza B, Storer BE, Sorror ML, Blume K, Niederwieser D, et al. Graft-versus-host disease and graft-versustumor effects after allogeneic hematopoietic cell transplantation. J Clin Oncol. 2013;31(12):1530-8. https://doi.org/10.1200/ jco.2012.45.0247 (Epub 2013 Mar 11, 2013).

6. Carlens S, Ringden O, Remberger M, Lönnqvist B, Hägglund $\mathrm{H}$, Klaesson S, et al. Risk factors for chronic graft-versus-host disease after bone marrow transplantation: a retrospective single centre analysis. Bone Marrow Transpl. 1998;22:755-61.

7. Ratanatharathorn V, Ayash L, Lazarus HM, Fu J, Uberti JP. Chronic graft-versus-host disease: clinical manifestation and therapy. Bone Marrow Transpl. 2001;28:121-9.

8. Atkinson K, Horowitz MM, Gale RP, van Bekkum DW, Gluckman E, Good RA, et al. Risk factors for chronic graft-versus-host disease after HLA-identical sibling bone marrow transplantation. Blood. 1990;75(12):2459-64.

9. Eapen M, Logan B, Confer D, Haagenson M, Wagner J, Weisdorf DJ, et al. Peripheral blood grafts from unrelated donors are associated with increased acute and chronic graft-versus-host disease without improved survival. Biol Blood Marrow Transpl. 2007;13:1461-8.

10. Perez-Simon JA, Afram G, Martino R, Pinana JL, CaballeroVelazquez T, Ringden O, et al. Evaluation of prognostic factors among patients with chronic graft-versus-host disease. Haematologica. 2012;97(8):1187-95.
11. Bacigalupo A, Lamparelli T, Barisione G, Bruzzi P, Guidi S, Alessandrino PE, et al. Thymoglobulin prevents chronic graft-versushost disease, chronic lung dysfunction, and late transplant-related mortality: long-term follow-up of a randomized trial in patients undergoing unrelated donor transplantation. Biol Blood Marrow Transpl. 2006;12(5):560-5.

12. Filipovich A, Weisdorf D, Pavletic S, Socie G, Wingard JR, Lee SJ, et al. National institutes of health consensus development project on criteria for clinical trials in chronic graft-versus-host disease: I. Diagnosis and staging working group report. Am Soc Blood Marrow Transpl. 2005;11:945-55.

13. Remberger M, Ringden O, Hägglund H, Svahn BM, Ljungman $\mathrm{P}$, Uhlin $\mathrm{M}$, et al. A high antithymocyte globulin dose increases the risk of relapse after reduced intensity conditioning HSCT with unrelated donors. Clin Transpl. 2013;27(4):E368-74. https ://doi.org/10.1111/ctr.12131 (Epub 2013 May 22, 2013).

14. Fine J, Gray R. Proportional hazard model for the sub-distribution of competing risks. J Am Stat Assoc. 1999;94:496-509.

15. Kolb HJ, Schmid C, Barrett AJ, Schendel DJ. Graft-versus-leukemia reactions in allogeneic chimeras. Blood. 2004;103(3):767-76 (Epub 2003 Sep 4, 2004).

16. Inamoto Y, Storer BE, Lee SJ, Carpenter PA, Sandmaier BM, Flowers ME, et al. Failure-free survival after second-line systemic treatment of chronic graft-versus-host disease. Blood. 2013;121(12):2340-6. https://doi.org/10.1182/blood-2012-11465583 (Epub 2013 Jan 15, 2013).

17. Lee SJ, Klein JP, Barrett AJ, Ringden O, Antin JH, Cahn JY, et al. Severity of chronic graft-versus-host disease: association with treatment-related mortality and relapse. Blood. 2002;100(2):406-14.

18. Saillard C, Crocchiolo R, Furst S, El-Cheikh J, Castagna L, Signori A, et al. National Institutes of Health classification for chronic graft-versus-host disease predicts outcome of allohematopoietic stem cell transplant after fludarabine-busulfanantithymocyte globulin conditioning regimen. Leuk Lymphoma. 2014;55(5):1106-12.

19. Couriel DR, Saliba RM, Giralt S, Khouri I, Andersson B, de Lima M, et al. Acute and chronic graft-versus-host disease after ablative and nonmyeloablative conditioning for allogeneic hematopoietic transplantation. BBMT. 2004;10:178-85.

20. Cremer B, Sandmaier BM, Bethge W, Lange T, Goede V, Holtick $\mathrm{U}$, et al. Reduced intensity conditioning in allogeneic stem cell transplantation for hematological malignancies: a historical perspective. Onkologie. 2011;34:710-5.

21. Flowers ME, Inamoto Y, Carpenter PA, Lee SJ, Kiem HP, Petersdorf EW, et al. Comparative analysis of risk factors for acute graft-versus-host disease and for chronic graft-versus-host disease according to National Institutes of Health consensus criteria. Blood. 2011;117(11):3214-9.

22. Kanda J, Nakasone H, Atsuta Y, Toubai T, Yokoyama H, Fukuda $\mathrm{T}$, et al. Risk factors and organ involvement of chronic GVHD in Japan. Bone Marrow Transpl. 2014;49(2):228-35. https://doi. org/10.1038/bmt.2013.151 (Epub 2013 Sep 30, 2013).

23. Mohty M, Bay JO, Faucher C, Choufi B, Bilger K, Tournilhac $\mathrm{O}$, et al. Graft-versus-host disease following allogeneic transplantation from HLA-identical sibling with antithymocyte globulin-based reduced-intensity preparative regimen. Blood. 2003;102(2):470-6 (Epub 2003 Mar 20, 2003).

24. Finke J, Bethge WA, Schmoor C, Ottinger HD, Stelljes M, Zander AR, et al. Standard graft-versus-host disease prophylaxis with or without anti-T-cell globulin in haematopoietic cell transplantation from matched unrelated donors: a randomised, openlabel, multicentre phase 3 trial. Lancet Oncol. 2009;10(9):85564. https://doi.org/10.1016/s1470-2045(09)70225-6 (Epub 2009 Aug 18, 2009). 
25. Pidala J, Tomblyn M, Nishihori T, Ayala E, Field T, Fernandez $\mathrm{H}$, et al. ATG prevents severe acute graft-versus-host disease in mismatched unrelated donor hematopoietic cell transplantation. Biol Blood Marrow Transpl. 2011;17(8):1237-44. https://doi. org/10.1016/j.bbmt.2010.12.705 (Epub 2011 Jan 6, 2011).

26. Langston AA, Prichard JM, Muppidi S, Nooka A, Lechowicz MJ, Lonial S, et al. Favorable impact of pre-transplant ATG on outcomes of reduced-intensity hematopoietic cell transplants from partially mismatched unrelated donors. Bone Marrow Transpl. 2014;49(2):185-9. https://doi.org/10.1038/ bmt.2013.168 (Epub 2013 Oct 28, 2014).

27. Slack JL, Dueck AC, Fauble VD, Sproat LO, Reeder CB, Noel P, et al. Reduced toxicity conditioning and allogeneic stem cell transplantation in adults using fludarabine, carmustine, melphalan, and antithymocyte globulin: outcomes depend on disease risk index but not age, comorbidity score, donor type, or human leukocyte antigen mismatch. Biol Blood Marrow Transpl. 2013;19(8):116774. https://doi.org/10.1016/j.bbmt.2013.05.001 (Epub 2013 May 7, 2013).

28. Russell JA, Turner AR, Larratt L, Chaudhry A, Morris D, Brown $\mathrm{C}$, et al. Adult recipients of matched related donor blood cell transplants given myeloablative regimens including pretransplant antithymocyte globulin have lower mortality related to graft-versus-host disease: a matched pair analysis. Biol Blood Marrow Transpl. 2007;13(3):299-306.

29. Bacigalupo A, Lamparelli T, Bruzzi B, Guidi S, Alessandrino PE, Bartolomeo P, et al. Antithymocyte globulin for graft-versus-host disease prophylaxis in transplants from unrelated donors: 2 randomized studies from Gruppo Italiano Trapianti Midollo Osseo (GITMO). Blood. 2001;98(10):2942-7.

30. Soiffer RJ, Lerademacher J, Ho V, Kan F, Artz A, Champlin RE, et al. Impact of immune modulation with anti-T-cell antibodies on the outcome of reduced-intensity allogeneic hematopoietic stem cell transplantation for hematologic malignancies. Blood. 2011;117(25):6963-70. https://doi.org/10.1182/blood-2011-01332007 (Epub 2011 Apr 4, 2011).

31. Devillier R, Crocchiolo R, Castagna L, Furst S, El Cheikh J, Faucher C, et al. The increase from 2.5 to $5 \mathrm{mg} / \mathrm{kg}$ of rabbit anti-thymocyte-globulin dose in reduced intensity conditioning reduces acute and chronic GVHD for patients with myeloid malignancies undergoing allo-SCT. Bone Marrow Transpl. 2012;47(5):639-45. https://doi.org/10.1038/bmt.2012.3 (Epub 2012 Feb 6, 2012).

32. Devillier R, Furst S, El-Cheikh J, Castagna L, Harbi S, Granata A, et al. Antithymocyte globulin in reduced-intensity conditioning regimen allows a high disease-free survival exempt of long-term chronic graft-versus-host disease. Biol Blood Marrow Transpl. 2013. https://doi.org/10.1016/j.bbmt.2013.11.030.

33. Sorror ML, Martin PJ, Storb RF, Bhatia S, Maziarz RT, Pulsipher MA, et al. Pretransplant comorbidities predict severity of acute graft-versus-host disease and subsequent mortality. Blood. 2014;124(2):287-95. https://doi.org/10.1182/blood-2014-01550566 (Epub 2014 May 5). 